\title{
Clustered Cooperative Communications in Wireless Networks
}

\author{
Ahmed K. Sadek, Weifeng Su*, and K. J. Ray Liu \\ Department of Electrical and Computer Engineering, and Institute for Systems Research \\ University of Maryland, College Park, MD 20742, USA. \{aksadek, kjrliu\}@eng.umd.edu \\ *Department of Electrical Engineering, State University of New York at Buffalo, USA. weifeng@eng.buffalo.edu
}

\begin{abstract}
In this paper, a cluster-based cooperative communication scheme is introduced in which each cluster acts as a virtual node with multiple antennas. The presented model considers the effects of random deployment of nodes and hence their random distribution across the network. Taking these effects into account, a pairwise-error probability analysis for a generic space-time code structure is provided. The analysis reveals that the diversity and coding gain of the system depends on both the code structure as well as the nodes' distribution, and hence new code design criteria are developed. Simulation results are presented to confirm the theoretical analysis.
\end{abstract}

\section{INTRODUCTION}

Cooperative communications in wireless networks have gained a lot of interest recently in the research community. Designing protocols that allow several single-antenna terminals to cooperate via forwarding each others data can increase the system reliability through achieving spatial diversity. Boosting the system throughput is another gain that we can benefit from employing cooperation between nodes in the network. Laneman et al. have proposed various cooperative diversity protocols in [1] and [2], and they provided outage probability performance. The concepts of decodeand-forward and amplify-and-forward have been introduced in these works. Sendonaris et al. introduced the concept of user-cooperation for CDMA systems in [3] and [4], where they considered cooperation between two users. Symbol error rate performance analysis for decode-and-forward single relay and multi-relay cooperative systems were provided in [5] and [6]. In [11], the authors emphasized the importance of studying distributed multistage relaying in which each stage acts as a virtual antenna, and they envision that this is a promising direction to achieve the very high data rates requirements of future wireless systems.

In this paper, we study the performance of cluster-based cooperative communications. Clustering in wireless networks means the aggregation of nodes into groups according to some criteria or features. Clustering is especially useful when we need to design scalable network functions in dense networks. Hence, in multihop mobile wireless networks clustering provides a convenient framework to design cooperation protocols. Nodes within the same cluster can cooperate forwarding information via forming virtual arrays.

\footnotetext{
${ }^{1}$ This work was supported in part by U.S. Army Research Laboratory under Cooperative Agreement DAAD 190120011.
}

Being in the same cluster, the delays between these nodes can be more easier to adjust in order to achieve synchronization. Space-time-coding, beamforming, spatial-multiplexing, and other conventional MIMO techniques can be extended and developed to suit this new framework. However, there are important technical issues that must be taken into account when studying this system. The nodes forming the virtual antennas are not as reliable as in the point-to-point communications context as the nodes can fail in decoding data due to wireless channel impairments. Another important issue is that due to random mobility of the nodes, the number of nodes within the cluster is random. Thus, we have a virtual array with a random number of antennas that are not all reliable. In this work, we introduce a cluster-based cooperative communications system taking into account the above issues. We analyze the system pairwise-error probability (PEP) for a generic space-time structure. We show that the unreliability of the nodes in the virtual array (some nodes may detect in error and thus remain idle during this frame transmission) does not affect the diversity gain of the system while it affects the coding gain. However, the randomness in the number of nodes in the cluster reduces the diversity order and we quantify the maximum diversity achieved in this case.

\section{Cooperation Protocol Model}

We consider a clustered wireless network in which cluster heads have been already selected and clusters are formed according to some criteria; for example cluster heads can be chosen to be the most powerful nodes in the network and clusters are formed to have certain transmission range $R$ that is pre-determined according to the clustering protocol (see [9] and references therein). This is a popular connectivity model in the literature of ad-hoc networks, however, it does not take into account the randomness in the wireless fading environment. In our analysis, we take this randomness into consideration, and the channel between any two nodes within the cluster is modeled as a Rayleigh fading channel contaminated with AWGN. For the node distribution in the network, we consider a two-dimensional Poisson process with intensity $\sigma$ as shown in [8], [10]. Hence, the probability of finding $k$ nodes in an area $A$ is given by

$$
P[K \text { nodes in } A]=\exp ^{-\sigma A} \frac{(\sigma A)^{k}}{k !} .
$$




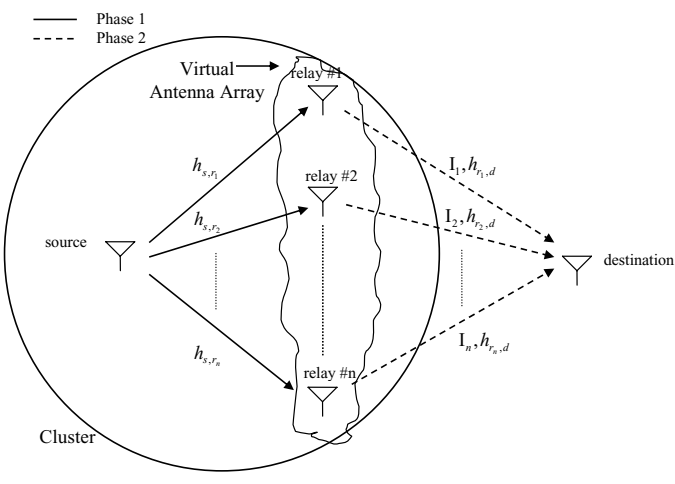

Fig. 1. A virtual array of size $n$-antennas formed within the cluster to help the source.

We want to develop a communication protocol that utilizes cooperation between nodes within the same cluster in order to form a virtual antenna array. Hence, we can consider clusters as a set of multiple antennas terminals that communicate with each other thus achieving higher transmission reliability via spatial diversity. We will consider a snapshot of this communications and try to analyze its performance to study the diversity and coding gain that can be achieved. If a node (source) needs to send information to a certain destination, a number of other nodes (relays) from the same cluster are assigned to help this source as depicted in Fig. 1. According to the communication protocol a maximum of $M$ nodes can be assigned to help the source. Hence, the number of nodes assigned to help the source is $\min (N, M)$, where $N$ is the number of nodes available in this cluster and follow the distribution in (1). Note that if $N=0$, it means the cluster-head itself helps the source transmit its information.

The relays assignment can be done by the cluster head, or in a distributed way. For example, the source sends a "Hello" message to nodes within the cluster, and then chooses from among these nodes which respond according to some selection criteria. Due to nodes mobility, the number of nodes associated with the cluster is varying, and the cluster head needs to update its information every time period. In practice, and due to slow speed of nodes velocity relative to the transmission speed of information, this time period can be more than one data-frame time.

Next, we describe the cooperation protocol which consists of two phases as depicted in Fig. 1. The number of relays cooperating with the source is a random variable denoted by $n$ and can take values between 1 and $M$ with probabilities

$$
P_{n}= \begin{cases}\frac{\left(\sigma A_{C}\right)^{n-1}}{(n-1) !} \exp ^{-\sigma A_{C}} & \text { if } 1 \leq n<M \\ \sum_{k=M-1}^{\infty} \frac{\left(\sigma A_{C}\right)^{k}}{k !} \exp ^{-\sigma A_{C}} & \text { if } n=M\end{cases}
$$

where $A_{C}$ is the cluster area, and $n=1$ if there are no nodes in the cluster other than the source and the cluster-head, which will forward the source's message in this case. In phase 1 , if $n$ relays are assigned for cooperation, the source transmits data to these relays with power $P_{1}$ and the signal received at the $i$-th relay can be modeled as

$$
\mathbf{y}_{s, r_{i}}=\sqrt{P_{1}} h_{s, r_{i}} \mathbf{s}+\mathbf{v}_{s, r_{i}}, \quad 1 \leq i \leq n
$$

where $\mathrm{s}$ is a $L_{n} \times 1\left(L_{n} \geq n\right)$ transmitted data vector with average energy $E\left[\|\mathbf{s}\|^{2}\right] \leq L_{n}$. $h_{s, r_{i}}$ denotes the channel gain between the source and the $i$-th relay and it follows a circularly symmetric complex Gaussian random process with zero mean and variance $\delta_{s, r}^{2}$ which we denote by $\mathcal{C N}\left(0, \delta_{s, r}^{2}\right)$. The channel gains from the source to the relays are assumed i.i.d. All channels are fixed during the transmission of one data packet and can vary from one packet to another. In (3), $\mathbf{v}_{s, r_{i}} \sim \mathcal{C N}\left(0, N_{o}\right)$ denotes additive white Gaussian noise AWGN. The $n$ relays tries to decode the received signals. We assume the transmitted data to be encoded with a cyclicredundance-check code (CRC) [7] such that the relays will be able to decide whether it decoded correctly or not. If a relay decodes correctly it will forward the data in the second phase of the cooperation protocol, otherwise it remains idle. The relays are assumed to be synchronized either through the cluster-head or by a distributed algorithm.

In phase 2 , the relays that decodes correctly re-encodes the data vector $\mathbf{s}$ with a pre-assigned code structure, which is assigned by the cluster head. In the subsequent development we do not assume a specific code design and we consider a generic space-time (ST) code structure. The ST code is distributed among the relays such that each relay will emulate a single antenna in a multiple antenna transmitter, and hence each relay will generate a column in the corresponding ST code matrix. Let $\mathbf{X}_{r_{i}}$ denote the $L_{n} \times 1$ code generated by the $i$-th relay. Hence the signal received at the destination from all relays can be modeled as

$$
\mathbf{Y}_{\mathbf{d}}=\sqrt{P_{2}}\left[I_{1} \mathbf{X}_{r_{1}} I_{2} \mathbf{X}_{r_{2}} \cdots I_{n} \mathbf{X}_{r_{n}}\right] \mathbf{h}_{d}+\mathbf{V}_{d}
$$

where $\mathbf{h}_{d}=\left[h_{r_{1}, d} h_{r_{2}, d} \cdots h_{r_{n}, d}\right]^{T}$ is an $n \times 1$ vector channel gains from the $n$ relays to the destination and $h_{r_{i}, d} \sim$ $\mathcal{C N}\left(0, \delta_{r, d}^{2}\right)$. The $n$ channels are assumed to be statistically independent as the relays are spatially separated. $\mathbf{V}_{d}$ is AWGN at the destination and has i.i.d. entries with zero mean and variance $N_{o}$. The state of the $k$-th relay, i.e., whether it decoded correctly or not, is denoted by the random variable $I_{k}(1 \leq k \leq n)$ which takes values 1 or 0 if the relay decodes correctly or erroneously, respectively. The random variables $I_{k}$ 's ( $\left.1 \leq k \leq n\right)$ are statistically independent as the state of each relay depends only on its channel conditions to the source which are independent from other relays.

There is an energy constraint on the generated ST code such as $E\left[\left\|\mathbf{X}_{\mathbf{r}}\right\|^{2}\right] \leq L_{n}$, where $\mathbf{X}_{\mathbf{r}}$ is an $L_{n} \times n \mathrm{ST}$ code matrix with the $k$-th column being the ST code vector $\mathbf{X}_{r_{k}}$ transmitted by the $k$-th relay if it decoded correctly. The 
total transmitted power is kept fixed as $P_{1}+P_{2}=P$ for fairness of comparison. Note that the number of relays $n$ cooperating with the source is a random variable governed by the distribution in (2).

\section{PERFormANCE ANALYSIS}

In this section, we provide pairwise error performance analysis for the cooperation scheme described in Section II. The diversity and coding gain achieved by the protocol are then analyzed.

\section{A. Pairwise Error Probability}

The received signal model at the destination in (4) can be rewritten as follows

$$
\mathbf{Y}_{\mathbf{d}}=\sqrt{P_{2}} \mathbf{X}_{\mathbf{r}} \mathbf{h}_{d, I}+\mathbf{V}_{d}
$$

The new channel definition $\mathbf{h}_{d, I}$ includes the information about both the channel realization from the $n$ relays to the destination along with the relay state information $\mathbf{I}=$ $\left[I_{1} I_{2} \cdots I_{n}\right]$ and is defined as follows

$$
\mathbf{h}_{d, I}=\left[I_{1} h_{r_{1}, d} I_{2} h_{r_{2}, d} \cdots I_{n} h_{r_{n}, d}\right]^{T} .
$$

Hence, if the $k$-th relay decoded in error then its equivalent channel $I_{k} h_{r_{k}, d}$ equals 0 .

Since the relay decides not to transmit if it decodes the received packet from the source in error, after checking with the CRC, the random variable $I_{k}$ is a Bernoulli random variable with a distribution given by

$$
I_{k}= \begin{cases}0 & \text { w.p. } \simeq L_{n} \text { SER } \\ 1 & \text { w.p. } \simeq 1-L_{n} \text { SER }\end{cases}
$$

where the probability of decoding a packet of length $L_{n}$ in error is approximated using the union-bound by $L_{n} \mathbf{S E R}$, where SER is the symbol error rate and is modulation dependent. For Q'ary quadrature amplitude modulation (QQAM, $Q=2^{k}$ with $k$ even), we can show that the exact expression can be upper bounded by

$$
\mathbf{S E R} \leq \frac{2 N_{o} g(2)}{b P_{1} \delta_{s, r}^{2}}
$$

where $b=3 /(Q-1)$ and $g(n)=\frac{4 K}{\pi} \int_{0}^{\pi / 2} \sin ^{2} \theta d \theta-$ $\frac{4 K^{2}}{\pi} \int_{0}^{\pi / 4} \sin ^{2} \theta d \theta$ in which $K=1-\frac{1}{\sqrt{Q}}$. The proof is omitted for lack of space.

The destination has full channel state information $\mathbf{h}_{d, I}$ and applies a maximum likelihood receiver which will be a minimum distance rule as follows

$$
\mathbf{X}=\arg \min _{\mathbf{X} \in \mathcal{X}}\left\|\mathbf{Y}_{\mathbf{d}}-\sqrt{P_{2}} \mathbf{X}_{\mathbf{r}} \mathbf{h}_{d, I}\right\|^{2},
$$

where $\mathcal{X}$ is the set of all possible codewords. Denote the conditional PEP that the destination decides the codeword $\hat{\mathbf{X}}$ when the codeword $\mathbf{X}$ was transmitted as $P(\mathbf{X} \rightarrow \hat{\mathbf{X}})$. We can show that the conditional PEP given the equivalent channel realization $\mathbf{h}_{d, I}$ and the number of relays $n$ can be upper bounded as follows

$$
P\left(\mathbf{X} \rightarrow \hat{\mathbf{X}} \mid \mathbf{h}_{d, I}, n\right) \leq \exp ^{-\frac{P_{2}\left\|\mathbf{\Phi}(\mathbf{X}, \hat{\mathbf{x}}) \mathbf{h}_{d, I}\right\|^{2}}{4 N_{o}}}
$$

where $\mathbf{\Phi}(\mathbf{X}, \hat{\mathbf{X}})=(\mathbf{X}-\hat{\mathbf{X}})$ is the difference matrix between the two codewords $\mathbf{X}$ and $\hat{\mathbf{X}}$. The Frobenius norm in (10) can be further decomposed as

$$
\left\|\boldsymbol{\Phi}(\mathbf{X}, \hat{\mathbf{X}}) \mathbf{h}_{d, I}\right\|^{2}=\mathbf{h}_{d}^{H} \mathbf{D}_{\mathbf{I}} \boldsymbol{\Phi}(\mathbf{X}, \hat{\mathbf{X}})^{H} \mathbf{\Phi}(\mathbf{X}, \hat{\mathbf{X}}) \mathbf{D}_{\mathbf{I}} \mathbf{h}_{d}
$$

where $\mathbf{D}_{\mathbf{I}}$ is an $n \times n$ diagonal matrix with the $k$-th diagonal entry equal to $I_{k}$. If the ST code structure is designed to achieve full diversity in the MIMO case, the difference matrix $\boldsymbol{\Phi}(\mathbf{X}, \hat{\mathbf{X}})$ is full rank. Hence the rank of the matrix $\boldsymbol{\Phi}(\mathbf{X}, \hat{\mathbf{X}}) \mathbf{D}_{\mathbf{I}}$, which is the difference code matrix after inserting zeros in the columns corresponding to the relays that decoded in error, equals to $\operatorname{rank}\left(\mathbf{D}_{\mathbf{I}}\right)$. This rank corresponds to the number of relays that decode correctly and in the sequel we refer to it by $r_{\mathbf{I}}$. The matrix $\mathbf{D}_{\mathbf{I}} \boldsymbol{\Phi}(\mathbf{X}, \hat{\mathbf{X}})^{H} \boldsymbol{\Phi}(\mathbf{X}, \hat{\mathbf{X}}) \mathbf{D}_{\mathbf{I}}$ is hermitian and can thus be decomposed into

$$
\mathbf{D}_{\mathbf{I}} \boldsymbol{\Phi}(\mathbf{X}, \hat{\mathbf{X}})^{H} \boldsymbol{\Phi}(\mathbf{X}, \hat{\mathbf{X}}) \mathbf{D}_{\mathbf{I}}=\mathbf{U} \boldsymbol{\Lambda} \mathbf{U}^{H},
$$

where $\mathbf{U}$ is a unitary matrix with the $i$-th column $\mathbf{u}_{i}$ denoting the $i$-th eigenvector and $\boldsymbol{\Lambda}$ is a diagonal matrix with the $i$-th entry $\lambda_{i}$ denoting the $i-t h$ eigenvalue which are arranged in a non-increasing order. We can write (11) using the decomposition in (12) as follows

$$
\mathbf{h}_{d}^{H} \mathbf{U} \boldsymbol{\Lambda} \mathbf{U}^{H} \mathbf{h}_{d}=\tilde{\mathbf{h}}_{d}^{H} \boldsymbol{\Lambda} \tilde{\mathbf{h}}_{d},
$$

where $\tilde{\mathbf{h}}_{d}=\mathbf{U}^{H} \mathbf{h}_{d}$. Since $h_{r_{i}, d}$ 's are independent with a Gaussian distribution, and the matrix $\mathbf{U}$ is unitary, then the elements of the vector $\tilde{\mathbf{h}}_{d}$ are also Gaussian and independent.

The random vectors $\tilde{\mathbf{h}}_{d}$ and $\mathbf{I}$ are mutually independent as they arise from independent processes $\left(\left\{h_{r_{i}, d}\right\}_{i=1}^{n}\right.$ are the channel realizations from the relays to the destination, while $\left\{I_{i}\right\}_{i=1}^{n}$ denote whether the $n$ relays decoded the information transmitted from the source correctly or not). Hence, we can average over these random variables separately. First, we average over the transformed channel realizations $\tilde{\mathbf{h}}_{d}$. The channel gain $\left|\tilde{h}_{d}(i)\right|^{2}(1 \leq i \leq n)$ has an exponential distribution with parameter $\left(1 / \delta_{r, d}^{2}\right)$. Averaging the conditional PEP in (10) over all channel realizations, we get

$$
P(\mathbf{X} \rightarrow \hat{\mathbf{X}} \mid I, n) \leq \prod_{i=1}^{r_{\mathbf{I}}} \frac{1}{1+\frac{P_{2} \delta_{r, d}^{2}}{4 N_{o}} \lambda_{i}} .
$$


Second, we average over different realizations of the relays states I. The dependence of the expression in (14) on I appears in the upper limit of the summation $r_{\mathbf{I}}$ and the set of non-zero eigenvalues $\left\{\lambda_{i}\right\}_{i=1}^{r_{\mathbf{I}}}$. The former quantity depends only on the number of relays that decode correctly, while the later quantity depends on both the number of relays that decoded correctly and their realization as this determines which columns from the ST code matrix are replaced with zeros and thus affect the resulting eigenvalues. To take into account the dependence of the set of non-zero eigenvalues $\left\{\lambda_{i}\right\}_{i=1}^{r_{I}}$ on the realization of the relays states $\mathbf{I}$, i.e. which relays decoded correctly, we upper bound the PEP in (14) as follows

$$
P(\mathbf{X} \rightarrow \hat{\mathbf{X}} \mid I) \leq \max _{\mathcal{I}} \prod_{i=1}^{r_{\mathbf{I}}} \frac{1}{1+\frac{P_{2} \delta_{r, d}^{2}}{4 N_{o}} \lambda_{i}},
$$

where $\mathcal{I}$ denotes the set of realizations $\mathbf{I}$ which have the same rank $r_{\mathbf{I}}$, i.e., has the same number of relays that decoded correctly. From another prospective, this upper bound corresponds to the minimum set of eigenvalues of the ST difference codewords after replacing $n-r_{\mathbf{I}}$ columns from them with zeros. We denote this set of eigenvalues by $\left\{\lambda_{i, r_{\mathbf{I}}}^{n}\right\}_{i=1}^{r_{\mathbf{I}}}$.

Since the $I_{k}$ 's are i.i.d. Bernoulli r.v.'s as in (7), the number of relays that decoded correctly $r_{\mathbf{I}}$ has a binomial distribution given by

$P_{r_{\mathbf{I}}}(k)=\left(\begin{array}{c}n \\ k\end{array}\right)\left(1-L_{n} \mathbf{S E R}\right)^{k}\left(L_{n} \mathbf{S E R}\right)^{n-k}, 0 \leq k \leq n$.

Averaging over all realizations of the states of the relays we get

$$
\begin{aligned}
P(\mathbf{X} \rightarrow \hat{\mathbf{X}} \mid n) \leq & \sum_{k=0}^{n}\left(\begin{array}{l}
n \\
k
\end{array}\right)\left(1-L_{n} \mathbf{S E R}\right)^{k}\left(L_{n} \mathbf{S E R}\right)^{n-k} \\
& \times \prod_{i=1}^{k}\left(1+\frac{P_{2} \delta_{r, d}^{2}}{4 N_{o}} \lambda_{i, k}^{n}\right)^{-1}
\end{aligned}
$$

Finally, since the number of relays available for helping the source is random due to the random deployment of the nodes in the network or due to random node mobility that causes nodes to come in and out of the cluster, the average PEP is given by

$$
\begin{aligned}
P(\mathbf{X} \rightarrow \hat{\mathbf{X}}) \leq & \sum_{n=1}^{M} P_{n} \sum_{k=0}^{n}\left(\begin{array}{l}
n \\
k
\end{array}\right)\left(1-L_{n} \mathbf{S E R}\right)^{k} \\
& \times\left(L_{n} \mathbf{S E R}\right)^{n-k} \prod_{i=1}^{k}\left(1+\frac{P_{2} \delta_{r, d}^{2}}{4 N_{o}} \lambda_{i, k}^{n}\right)^{-1},
\end{aligned}
$$

where $P_{n}$ is defined in (2), and $M$ is the maximum number of relays that can be assigned to help a source.

\section{B. Diversity and Coding Gain of the system}

The diversity order of a system determines the average rate with which the error probability decays at high enough signal to noise ratio (SNR). In order to compute the diversity order of our system, we rewrite the PEP in (18) in terms of the SNR defined as $S N R=P / N_{o}$, where $P$ is the total power used in transmission from the source and the relays. Let the ratios of the total power assigned to the source and the relays be denoted by $a_{1}$ and $a_{2}$ respectively, where $P_{1}=a_{1} P$ and $P_{2}=a_{2} P$. Substituting these definitions along with the SER expression at the relays from (8) into (18) we get

$$
\begin{aligned}
P(\mathbf{X} \rightarrow \hat{\mathbf{X}}) \leq \sum_{n=1}^{M} & P_{n} S N R^{-n} \sum_{k=0}^{n}\left(\begin{array}{l}
n \\
k
\end{array}\right)\left(\frac{2 L_{n} g(2)}{b a_{1} \delta_{s, r}^{2}}\right)^{n-k} \\
& \times \prod_{i=1}^{k}\left(\frac{a_{2} \delta_{r, d}^{2}}{4} \lambda_{i, k}^{n}\right)^{-1},
\end{aligned}
$$

where at high $\mathrm{SNR}$ we can approximate $1-L_{n} \mathbf{S E R} \simeq 1$ and ignore the 1 term in the denominator added to the signal to noise ratio from the relay to the destination. The diversity gain achieved for a fixed network realization (fixed number of cooperating nodes) can be defined as $d=\lim _{S N R \rightarrow \infty}-$ $\frac{\log (P E P(S N R))}{\log (S N R)}$, where $P E P$ is the PEP. Applying this definition to the conditional PEP when the number of cooperating nodes is $n$ (17) we get

$$
d_{n}=\lim _{S N R \rightarrow \infty}-\frac{\log \left(C_{n} S N R^{-n}\right)}{\log (S N R)}=n
$$

where $C_{n}=\sum_{k=0}^{n}\left(\begin{array}{l}n \\ k\end{array}\right)\left(\frac{2 L_{n} g(2)}{b a_{1} \delta_{s, r}^{2}}\right)^{n-k} \prod_{i=1}^{k}\left(\frac{4}{a_{2} \delta_{r, d}^{2} \lambda_{i, k}^{n}}\right)$ is a term that does not depend on the SNR. From (20), for a fixed number of cooperating terminals $n$ the cooperation protocol can achieve full diversity gain. However, this diversity is random as it depends on the number of relays available for cooperation, and thus the total achieved diversity of the system is given by

$$
d=\sum_{n=1}^{M} P_{n} d_{n}=\sum_{n=1}^{M} P_{n} n,
$$

and it depends on the node distribution density. We will verify this effect in the simulations.

The coding gain can be found in a similar way. The coding gain is the factor that multiplies the SNR in the PEP expression. For a fixed network realization (fixed number of antennas $n$ ), the PEP in (17) can be written as

$$
P(\mathbf{X} \rightarrow \hat{\mathbf{X}} \mid n) \leq\left(C_{n}^{-1 / n} S N R\right)^{-n},
$$

where $C_{n}$ is as defined before. The total coding gain of the system averaged over all possible network configurations is 


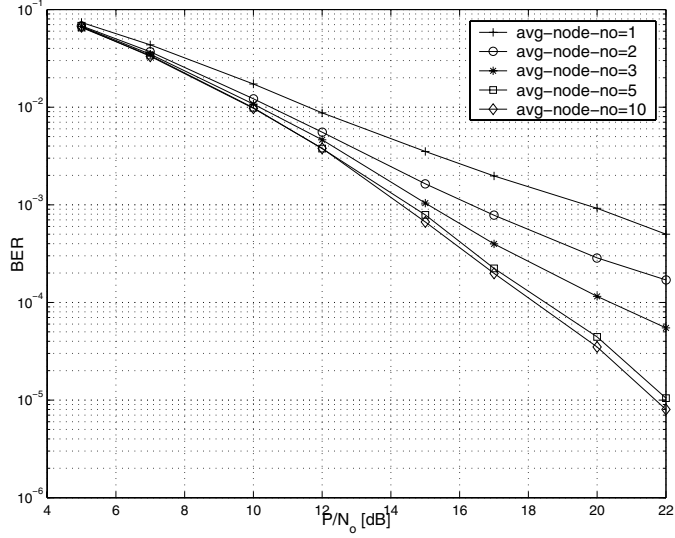

Fig. 2. BER performance of the clustered cooperation protocol with $M=$ 3 and different node densities.

given by

$$
\begin{gathered}
\Delta=\sum_{n=1}^{M} P_{n} C_{n}^{-1 / n} \\
C_{n}=\sum_{k=0}^{n}\left(\begin{array}{l}
n \\
k
\end{array}\right)\left(\frac{2 L_{n} g(2)}{b a_{1} \delta_{s, r}^{2}}\right)^{n-k} \prod_{i=1}^{k}\left(\frac{4}{a_{2} \delta_{r, d}^{2} \lambda_{i, k}^{n}}\right) .
\end{gathered}
$$

The coding gain of the system (23) is a function of the eigenvalues of the ST codewords after deleting different columns from it (corresponding to relays failure), and hence the design should take this into consideration by providing uniformly distributed eigenvalues for the ST codes that are robust to such relay failure.

\section{Simulation Results}

In this section, we provide computer simulations to study the effect of node distribution on the system performance. Since the PEP analysis derived in the paper is for a generic ST code structure, the results applies for any specific code. We chose the diagonal algebraic ST codes [12] to run the simulations. The maximum number of relays $M$ that can be assigned to any source is set to 3 in all simulations. In the case that only one relay is available for cooperation, the relays repeats the source symbol if it decodes correctly otherwise it remains idle. The channel variance between the source and any relay is set to 10 while that from the relay to the destination is set to 1 , and the noise variance $N_{o}=1$. BPSK modulation is used at the source node. Fig. 2 depicts the BER performance against $P / N_{o}$ for different average number of nodes per cluster $\left(\sigma A_{C}\right)$. Increasing $\sigma A_{C}$ increases the probability of finding more nodes in the cluster and hence increases the probability that $M=3$. This increases the maximum achievable diversity given in (21) and thus the system performance. It is clear from the figure that for $\sigma A_{C}=1,2$, and 3 , the diversity gain increases but diversity order $M=3$ can not be achieved. For $\sigma A_{C}=5$ and 10 the diversity gain almost approaches 3 that is why the improvement in the performance almost saturates. This is because as full diversity is achieved, the performance is only limited by the erroneous decoding of the relays which depends solely on the channel randomness and not the node distribution. This confirms our theoretical analysis.

\section{CONCLUSION}

We introduced a cluster-based cooperative communication scheme utilizing distributed ST coding. The clusters act like virtual arrays with random number of antennas due to the random deployment or mobility of nodes across the network. Our pairwise error probability analysis for a generic ST coding structure under this network model reveals that new code design criteria arises that should be taken into consideration for this new communication paradigm. For a fixed number of cooperating relays, the cooperation protocol achieves full diversity, however, the average achieved diversity gain is affected by the node distribution. The coding gain is affected by both the node failure due to erroneous decoding and the node distribution. Simulation results confirm our theoretical analysis.

\section{REFERENCES}

[1] J. N. Laneman and G. W. Wornell, "Distributed space-time coded protocols for exploiting cooperative diversity in wireless networks," IEEE Trans. Inform. Theory, vol. 49, pp.2415-2525, Oct. 2003.

[2] J. N. Laneman, D. N. C. Tse, and G. W. Wornell, "Cooperative diversity in wireless networks: efficient protocols and outage behavior," IEEE Trans. Inform. Theory, vol. 50, pp.3062-3080, Dec. 2004.

[3] A. Sendonaris, E. Erkip, and B. Aazhang, "User cooperation diversityPart I: system description,” IEEE Trans. Comm., vol. 51, pp.1927-1938, Nov. 2003.

[4] A. Sendonaris, E. Erkip, and B. Aazhang, "User cooperation diversityPart II: implementation aspects and performance analysis," IEEE Trans. Comm., vol. 51, pp.1939-1948, Nov. 2003.

[5] W. Su, A. K. Sadek, and K. J. R. Liu, "SER performance analysis and optimum power allocation for decode-and-forward cooperation protocol in wireless networks," to appear in IEEE Wireless Communications and Networking Conference (WCNC'05), New Orleans, LA, March 1317, 2005.

[6] A. K. Sadek, W. Su, and K. J. R. Liu, "Performance analysis for multinode decode-and-forward relaying in collaborative wireless networks," to appear in IEEE International Conference on Acoustics, Speech, and Signal Processing (ICASSP), Philadelphia, PA, March 19-23, 2005.

[7] P. Merkey and E. C. Posner, "Optimum cyclic redundancy codes for noise channels," IEEE Trans. Inform. Theory, vol. IT-30, pp..865-867, Nov. 1984.

[8] P. Samar and S. B. Wicker, "On the behavior of communication links of a node in a multi-hop- mobile environment," MobiHoc 2004, May 24-26, Roppongi, Japan.

[9] O. Younis and S. Fahmy, "Distributed clustering in ad-hoc sensor networks: A hybrid, energy-efficient approach," in Proceedings of IEEE INFOCOM, March 2004.

[10] M. Haenggi, "On routing in random Rayleigh fading networks", IEEE Trans. on Wireless Communications, accepted for publications, 2004.

[11] R. Pabst et al., "Relay-based deployment concepts for wireless and mobile broadband radio", IEEE Communications Magazine, vol. 42, no. 9 , pp. 80-89, Sep. 2004.

[12] M. O. Damen, K. Abed-Meraim, and J.C. Belfiore, "Diagonal algebraic spacetime block codes", IEEE Trans. on Information Theory, vol. 48, no. 3, pp. 628-636, March 2002. 